\title{
Selection based inter-relay power allocation for multiple AF single-antenna relays
}

Youngtaek Bae and Jungwoo Lee*

\begin{abstract}
This article deals with inter-relay power allocation for multiple-input multiple-output systems with multiple singleantenna relays. It is difficult to derive an optimal solution in a closed form for the case of multiple multi-antenna relays as well as the case of multiple single-antenna relays. In this article, we propose an approximate solution which is effective only for the case of multiple single-antenna relays. A key contribution of this article is a low complexity inter-relay power allocation method which is based on relay selection. This approach also reduces the feedback information of the gain factors of all the relays. An incremental greedy search algorithm is also proposed to further reduce the complexity of the relay selection process with negligible performance degradation.

Simulations indicate that the performance is comparable to the optimal exhaustive search algorithm.
\end{abstract}

\section{Introduction}

The relay channel was first studied by Meulen [1] and Cover and Gamal [2], which ignited research interest on the relay channel. Using a relay node, the transmission coverage can be extended with low cost, and a source node can transmit the signal more reliably through increased diversity. These advantages come from the cooperation between the source, the geographically distributed relays, and well developed combining techniques at the destination. Many articles have considered different forwarding schemes at the relay node to use the system resource efficiently $[3,4]$. They are usually classified by regenerative and non-regenerative methods depending on whether or not the source signal is regenerated at the relay node. The well known decode-andforward (DF) and amplify-and-forward (AF) schemes are examples of the two cases [5]. This general area is called cooperative communication, but some authors call it user cooperation by considering the case where each user can act as a source and a relay [6].

Power allocation methods for cooperative relay channel have been considered in $[7,8]$. However they all focused on the scenario of three nodes, each of which is equipped with a single antenna. The solutions can be obtained with a relatively straightforward manner. Recently, the scenario was extended to two different

\footnotetext{
* Correspondence: junglee@snu.ac.kr

School of Electrical Engineering, Seoul National University, Seoul, Korea
}

cases. One is the scenario of multiple relay nodes with a single antenna. In this case, the optimal power allocation has been derived in $[9,10]$. The other is the scenario of a single relay with multiple antennas while there are still three nodes in the entire system, and the source and the destination also have multiple antenna. The optimal power allocation for the latter case was proposed in $[11,12]$, which minimizes mean square error (MSE). Pairwise error probability (PEP) using distributed space-time codes was investigated in [13]. Overall channel capacity was considered in $[14,15]$. They all compute the singular value decomposition of the first hop and the second hop channels, and distribute the total power among the eigenmodes to optimize the performance criteria, MSE or channel capacity.

Recently the most general extension, i.e., a multipleantenna source and a multiple-antenna destination aided by multiple relays with multiple antennas, is considered in $[16,17]$. They computed the precoding matrix which is used for forwarding in the second hop. In [16], even though the proposed approach has a closed form for a diagonal matrix denoted by $\boldsymbol{\Phi}$, the final relay precoding matrix should be chosen in order to be a block diagonal matrix with minimum norm. That is, the solution does not have a closed form. Several relaying schemes for each relay are proposed in [17], but they are not capacity-optimal solutions. In $[18,19]$, the authors considered a similar system model with relaying through distributed spacetime codes, and showed that the power allocation solution 
results in the best relay selection. In fact, it is difficult to obtain a closed form solution for this general case with the block diagonal constraint. As a simpler case, if multiple relays with a single antenna are distributed geographically in a network, the matrix formed by all relay gains should be a diagonal matrix when full cooperation is not possible. However, few related results exist even for this simple case. Recently, [20] considered this simple system model, and derive an asymptotical capacity scaling law. However, they did not deal with the relay power allocation problem as in this article. Thus we focus on this simplified system where there are multiple relays with a single antenna while the source and the destination have multiple antennas. This may also be a practical scenario in the sense that the computational complexity of the relay nodes needs to be minimized. In order to focus on the effect of relay power gain, and compare with the performance of conventional non-regenerative relaying schemes, we only consider the AF scheme and real valued power gain ${ }^{\mathrm{a}}$ in this article. For the aforementioned scenario, we propose a relay selection based approach as an approximation to solve for the power allocation problem. It will be shown by simulations that the performance of the proposed method is close to that of the exhaustive (optimal) search approach. We then apply an incremental greedy search algorithm to this proposed method in order to further reduce the computational complexity of the selection based approach. This article deals only with the AF scheme, but a similar approach can be applied to the DF scheme with a modified object function instead of channel capacity.

The remainder of the article is organized as follows. In Section 2, we describe the system model and formulate the problem. We consider the existing inter-relay power allocation in Section 3, and propose the relay selection based approach for capacity maximization in Section 4. In Section 5, we discuss the feedback overhead and the computational complexity. Simulation results are given in Section 6 to assess the performance of the proposed method. Finally, we provide the conclusion in Section 7.

Notation: Boldface lowercase and uppercase letters represent vectors and matrices, respectively. $(.)^{*}$ denotes the complex conjugate transpose of a matrix. $|\cdot|$ means the determinant or the Euclidean $\left(l_{2^{-}}\right)$norm depending on the operand (a matrix or a vector).

\section{System model and problem formulation}

The source node and the destination node have $M$ transmit antennas and $N$ receive antennas, respectively. In the network, there are $R$ relays with a single antenna. To satisfy the rank requirement of a multiplexing system, we assume $M \leq N$ and $M \leq R$. However, we do not constrain the relationship between $R$ and $N$. First, the source broadcasts $M$ symbols to all relays in the first hop, and each relay multiplies the received noisy signal with a power gain. After that, all relays transmit these signals to the destination simultaneously in the second hop assuming perfect synchronization among relays. As depicted in Figure 1, the equivalent discrete time signal model can be summarized as follows,

$$
\begin{aligned}
\mathbf{y}_{r} & =\mathbf{H}_{1} \mathbf{x}+\mathbf{n}_{1} \\
\mathbf{y}_{d} & =\mathbf{H}_{2} \mathbf{x}_{r}+\mathbf{n}_{2}=\mathbf{H}_{2} \mathbf{G} \mathbf{H}_{1} \mathbf{x}+\mathbf{H}_{2} \mathbf{G} \mathbf{n}_{1}+\mathbf{n}_{2} \\
& \triangleq \mathbf{H x}+\mathbf{n},
\end{aligned}
$$

where $\mathbf{x}$ is the $(M \times 1)$ transmitted signal vector from the source node with covariance matrix $\mathbf{R}_{\mathbf{x}}$, and $\mathbf{y}_{r}(R \times$ $1)$ and $\mathbf{y}_{d}(N \times 1)$ denote the received signal at all the relays and the destination. $\mathbf{H}_{1}$ and $\mathbf{H}_{2}$ are $(R \times M)$ and $(N \times R)$ channel coefficient matrices from the source to all the relays, and from all the relays to the destination, which have independent and complex Gaussian distributed elements with zero mean and unit variance, i.e., Rayleigh fading. We assume that the source-relay and the relay-destination channels have an identical Rayleigh distribution, which means that all the relays are equidistant from the source and the destination. $\mathbf{n}_{1}$ and $\mathbf{n}_{2}$ are additive complex Gaussian noise with covariance matrices $\mathbf{R n}_{1}$ and $\mathbf{R} \mathbf{n}_{2}$, and $\mathbf{G}$ is a diagonal matrix made by relay power gains $\mathbf{g}=\left(g_{1}, \ldots, g_{R}\right) \cdot \mathbf{H}=\mathbf{H}_{2} \mathbf{G H}_{1}$ is the equivalent channel matrix, $\mathbf{n}=\mathbf{H}_{2} \mathbf{G} \mathbf{n}_{1}+\mathbf{n}_{2}$ is the compounded noise vector with covariance $\mathbf{R}_{\mathbf{n}}=\mathbf{H}_{2} \mathbf{G R}_{\mathbf{n}_{1}} \mathbf{G}^{*} \mathbf{H}_{2}^{*}+\mathbf{R}_{\mathbf{n}_{2}}$.

The general capacity formula or the mutual information can be written as in [21]

$$
\begin{aligned}
I\left(\mathbf{x} ; \mathbf{y}_{d}\right)=\frac{1}{2} & \log _{2}\left|\mathbf{I}_{M}+\mathbf{R}_{\mathbf{x}} \mathbf{H}^{*} \mathbf{R}_{n}^{-1} \mathbf{H}\right| \\
=\frac{1}{2} \log _{2} \mid \mathbf{I}_{M} & +\mathbf{R}_{\mathbf{x}} \mathbf{H}_{1}^{*} \mathbf{G}^{*} \mathbf{H}_{2}^{*} \\
& \times\left(\mathbf{H}_{2} \mathbf{G R}_{\mathbf{n}_{1}} \mathbf{G}^{*} \mathbf{H}_{2}^{*}+\mathbf{R}_{\mathbf{n}_{2}}\right)^{-1} \mathbf{H}_{2} \mathbf{G} \mathbf{H}_{1} \mid,
\end{aligned}
$$

where the factor $1 / 2$ is multiplied because we use two hops to transmit the signal. Our goal is to maximize this value with the individual power constraint allocated on each relay, $g_{k}^{2} \leq\left(g_{k}^{2}\right)^{\text {max }}$, where $\left(g_{k}^{2}\right)^{\text {max }}$ is the maximum value of the $k$ th relay power. In addition, there is the average power constraint on the relay output such

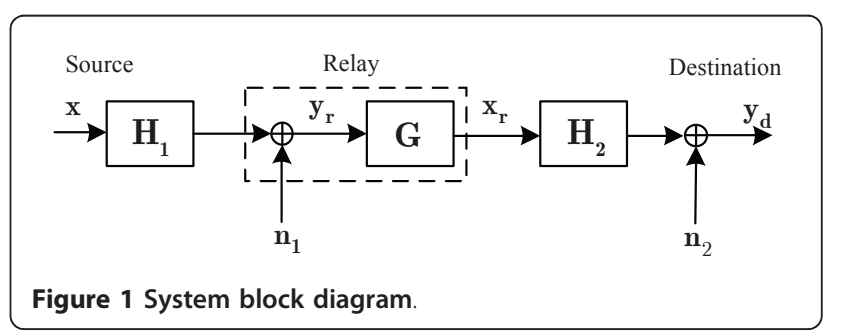


as $\operatorname{tr}\left\{\mathbf{R}_{\mathbf{x}_{r}}\right\} \leq E_{r}$, where $\mathbf{R}_{\mathbf{x}_{\mathrm{r}}}=\mathbf{G H}_{1} \mathbf{R}_{\mathbf{x}} \mathbf{H}_{1}^{*} \mathbf{G}^{*}+\mathbf{G R}_{\mathbf{n}_{1}} \mathbf{G}^{*}$ and $E_{r}$ is the total power of all the relays.

Problem formulation:

$$
\begin{array}{ll}
\operatorname{maximize} & I\left(\mathbf{x} ; \mathbf{y}_{d}\right) \\
\text { subject to } 0 \leq g_{k}^{2} \leq\left(g_{k}^{2}\right)^{\max }, \quad k=1, \ldots, R \\
\operatorname{tr}\left\{\mathbf{G H}_{1} \mathbf{R}_{\mathbf{x}} \mathbf{H}_{1}^{*} \mathbf{G}^{*}+\mathbf{G R}_{\mathbf{n}_{1}} \mathbf{G}^{*}\right\} \leq E_{r} .
\end{array}
$$

In the objective function, the diagonal matrix $\mathbf{G}$ depends on the first hop and the second hop channels, so it is difficult to obtain a closed form solution.

\section{Inter-relay power allocation}

Since the problem may have no closed form solution, we try to find a solution for optimal power allocation by numerical search. Power allocation is performed at the destination node, and the power gain values are fed back to all the relays. We assume that the destination node knows all the channel state information. We also assume $\mathbf{R}_{\mathbf{x}}=\frac{E_{s}}{M} \mathbf{I}_{M}$, where $E_{s}$ is the average transmit power, $\quad \mathbf{R}_{\mathbf{n}_{1}}=\sigma_{R}^{2} \mathbf{I}_{R}$, and $\mathbf{R}_{\mathbf{n}_{2}}=\sigma_{D}^{2} \mathbf{I}_{N}$. Since $\mathbf{I}_{M}+\mathbf{R}_{\mathbf{X}} \mathbf{H}^{*} \mathbf{R}_{n}^{-1} \mathbf{H}$ is a positive definite matrix, the eigenvalues of this matrix and the determinant in the capacity formula have bounded real values.

\subsection{Uniform}

A simple method is to use a uniform gain across all the relays. If the values of $g_{k}$ are a constant $(\beta)$, we can set $\mathbf{G}=$ $\beta \mathbf{I}$ where $\beta$ is chosen to satisfy the constraints. In this case, the capacity can reach the maximum when the equality of the constraint holds. From the average power constraint,

$$
\beta^{2}=\frac{E_{r}}{\sum_{k=1}^{R}\left(\frac{E_{s}}{M}\left|\mathbf{h}_{1 k}^{T}\right|^{2}+\sigma_{R}^{2}\right)},
$$

where $\mathbf{h}_{1 k}^{T}$ means the $k$ th row vector of $\mathbf{H}_{1}$. From the individual power constraint, this value should be less than or equal to $\left(g_{k}\right)^{\max }$ for each $k$. Therefore,

$$
g_{k}^{\text {Unif. }}=\left[\sqrt{\frac{E_{r}}{\sum_{k=1}^{R}\left(\frac{E_{s}}{M}\left|\mathbf{h}_{1 k}^{T}\right|^{2}+\sigma_{R}^{2}\right)}}\right]_{0}^{\left(g_{k}\right)^{\max }},
$$

where we use the notation $[x]_{l}^{u}=\max (l, \min (x, u))$. If the individual constraint is sufficiently large, all $g_{k}$ 's will be the same as $\beta$.

\subsection{Conventional power allocation}

In [4], the authors proposed a so called conventional power gain. It is given by the following equation

$$
g_{k}^{\text {Conv. }}=\left[\sqrt{\frac{E_{r} / R}{\frac{E_{s}}{M}\left|\mathbf{h}_{1 k}^{T}\right|^{2}+\sigma_{R}^{2}}}\right]_{0}^{(g k)^{\max }} .
$$

It should be noted that the conventional power allocation depends only on its own first hop channel while the uniform gain allocation depends on the first hop channels of all the relays.

\section{Proposed relay selection based approach}

The utility function to be maximized is the determinant of $\mathbf{K}=\mathbf{I}_{M}+\mathbf{R}_{\mathbf{x}} \mathbf{H}^{*} \mathbf{R}_{n}^{-1} \mathbf{H}$. substituting the equivalent channel and the compounded noise covariance, it is given by

$$
\begin{aligned}
& \mathbf{K}=\mathbf{I}_{M}+\frac{E_{s}}{M} \mathbf{H}_{1}^{*} \mathbf{G}^{*} \mathbf{H}_{2}^{*}\left(\sigma_{D}^{2} \mathbf{I}_{N}+\sigma_{R}^{2} \mathbf{H}_{2} \mathbf{G G}^{*} \mathbf{H}_{2}^{*}\right)^{-1} \mathbf{H}_{2} \mathbf{G H}_{1},(7) \\
& =\mathbf{I}_{M}+\frac{E_{s}}{M}\left(\sum_{i=1}^{R} g_{i} h_{2 i} h_{1 i}^{T}\right)^{*}\left(\sigma_{D}^{2} \mathbf{I}_{N}+\sigma_{R}^{2} \sum_{j=1}^{R} g_{j}^{2} h_{2 j} h_{2 j}^{*}\right)^{-1}\left(\sum_{k=1}^{R} g_{k} h_{2 k} h_{1 k}^{T}\right)
\end{aligned}
$$

where $g_{i}$ is the $i$ th diagonal element of $\mathbf{G}, h_{2 i}$ is the $i$ th column of $\mathbf{H}_{2}$, and $h_{1 i}^{T}$ is the ith row of $\mathbf{H}_{1}$.

It was observed that the optimal solution for $\mathbf{G}$ may never use certain relays in a poor channel condition, which motivates us to develop a relay selection based approach. In the selection based approach, the solution can be found by

$$
\max _{\mathbf{g}}|\mathbf{K}|=\max _{\mathbf{r} \subset\{1, \ldots, R\}} \max _{\mathrm{g}_{\mathrm{r}}}\left|\mathbf{K}\left(\mathrm{g}_{\mathrm{r}}\right)\right|,
$$

where $\mathbf{r}$ is a subset of relays, and $\mathbf{g}_{\mathbf{r}}$ is a vector with zeros at the position of $\mathbf{r}^{C}$. For example, if $R=5$ and $\mathbf{r}$ $=\left[\begin{array}{lll}1 & 2 & 4\end{array}\right], \mathbf{g}_{\mathbf{r}}=\left[g_{1}, g_{2}, 0, g_{4}, 0\right]$. To reduce the search complexity, the optimization over $\mathbf{g}_{\mathbf{r}}$ can be skipped. A sub-optimal solution can be obtained by finding a solution for the lower bound of the original problem as

$$
\max _{\mathbf{g}}|\mathbf{K}| \geq \max _{\mathbf{r} \subset\{1, \ldots, R\}}\left|\mathbf{K}\left(\mathbf{g}_{\mathbf{r}}^{\text {Unif. }}\right)\right|,
$$

where $\mathbf{g}_{\mathbf{r}}^{\text {Unif. }}$ denotes the gain vector for uniform gain allocation. The solution is obtained as if only the relays indicated by $\mathbf{r}$ participates in the relaying step. In (10), we have the inequality because the optimization over $\mathbf{g}_{\mathbf{r}}$ is skipped. The approach in (10) turns out to be a relay selection technique with uniform gain allocation among only selected relays to fully satisfy the original constraint. We chose to use the uniform gain allocation instead of the conventional power allocation because the former has better performance than the latter in the terms of capacity, which will be discussed in the simulation section. As will be shown in the simulation results, 
even with the sub-optimal approach which maximizes the lower bound, the proposed method can achieve performance similar to the optimal solution obtained by exhaustive search. The proposed approach obviously has lower complexity than the exhaustive search.

\section{Feedback requirement and computational complexity}

\subsection{Feedback requirement}

Let us consider the required amount of feedback bits from the destination to the relays. In the optimal solution by the exhaustive search, the power gain values need to be fed back to the corresponding relays. To use uniform gain allocation, we need to feed back the common power gain $\beta$ to the relays, which can be broadcasted by the destination. The conventional power allocation depends only on its own first hop channel from the source to each relay which is assumed available at the relay. Thus relay power can be calculated in each relay. Assuming that the total power $E_{r}$ and the number of relays $R$ are known at the relays, there is no need for feedback in this scheme.

The relay selection based approach needs to feed back the selection indicator bits as well as the power gain values of the selected relays. As for the selection indicator bits, one bit ( 0 or 1$)$ for each relay can be used to indicate whether a given relay is selected or not. Note that the feedback overhead of the proposed method will be significantly less than that of the optimal exhaustive search. When uniform gain allocation is used with the relay selection, only a single value (the common power gain) can be broadcasted by the destination. If conventional power allocation is used with the relay selection, the number of selected relays need to be broadcasted by the destination. As mentioned before, we employ uniform gain allocation in this article because it has slightly better performance than the conventional power allocation when no relay selection is used, which will be shown in the simulation results.

\subsection{Computational complexity}

The full search solution of proposed method gives us information on which relays are selected. However, the full search needs the computation of the utility function for every possible relay subset, and comparison of these values. When the number of relays $R$ is large, the number of possible relay subsets is

$$
\sum_{i=1}^{R}\left(\begin{array}{c}
R \\
i
\end{array}\right)=2^{R}-1
$$

which increases exponentially as $R$ increases, $O\left(2^{R}\right)$. Thus the complexity of the selection process itself may become infeasible. In order to reduce this selection complexity, we use an incremental greedy search algorithm. This algorithm first selects the relay with maximum utility function assuming that there is only one relay in the network. At the second stage, the algorithm searches for another relay (among the remaining $R-1$ relays) which maximizes the utility function when combined with previously selected relay. These operations are repeated until the utility function decreases or all the relays are selected. This greedy algorithm is summarized as in Algorithm 1. We use the notations where $\mathbf{H}_{1}([\mathcal{S}, n],:)$ and $\mathbf{H}_{2}(:,[\mathcal{S}, n])$ denote the matrices containing only row vectors or column vectors corresponding to index $[\mathcal{S}, n]$ in $\mathbf{H}_{1}$ and $\mathbf{H}_{2}$ where $\mathcal{S}$ is the set of pre-selected relays, and $n$ is the index for a candidate relay.

\section{Algorithm 1 Greedy search algorithm of the pro- posed method \\ Initialization:

$$
\mathcal{T}=\{1,2, \ldots, R\} ; \quad \mathcal{S}=\emptyset ; \quad f_{\max }=0 \quad m=0 ;
$$

Iteration:

$m \Re m+1$

$F O R n=1$ to $R$

IF $(n \in \mathcal{T})$

$$
\begin{aligned}
& \tilde{\mathbf{H}}_{1}=\mathbf{H}_{1}([\mathcal{S}, n],:) ; \quad \tilde{\mathbf{H}}_{2} \\
& \tilde{\mathbf{G}}=\sqrt{\frac{E_{r}}{\operatorname{tr}\left[\frac{E_{s}}{M} \tilde{\mathbf{H}}_{1} \tilde{\mathbf{H}}_{1}^{*}+\sigma_{R}^{2} \mathbf{I}_{m}\right]}} \mathbf{I}_{m} ; \\
& \mathbf{K}_{n}=\mathbf{I}_{M}+\frac{E_{s}}{M} \tilde{\mathbf{H}}_{1}^{*} \tilde{\mathbf{G}}^{*} \tilde{\mathbf{H}}_{2}^{*}\left(\sigma_{D}^{2} \mathbf{I}_{N}+\sigma_{R}^{2} \tilde{\mathbf{H}}_{2} \tilde{\mathbf{G}} \tilde{\mathbf{G}} \tilde{\mathbf{H}}_{2}^{*} \tilde{\mathbf{H}}^{*}\right)^{-1} \tilde{\mathbf{H}}_{2} \tilde{\mathbf{G}} \tilde{\mathbf{H}}_{1} ;
\end{aligned}
$$

END

$$
\begin{aligned}
& E N D^{p=\underset{n \in \mathcal{T}}{\arg \max }\left|\mathbf{K}_{n}\right| ;} \\
& I F\left(\left|\mathbf{K}_{p}\right|>f_{\max }\right)
\end{aligned}
$$

$\mathcal{T}=\mathcal{T}-\{p\} ; \mathcal{S}=\mathcal{S}+\{p\} ; f_{\max }=\left|\mathbf{K}_{p}\right| ;$

Go to Iteration:

\section{ELSE}

\section{Stop Iteration;}

\section{END}

From simulations, it was observed that the greedy algorithm usually stops at a value much smaller than $R$. Even in the worst case where the iteration continues until $m=R$, this only requires $\frac{R(R+1)}{2}$ computations and comparisons which have quadratic growth rate, $O\left(R^{2}\right)$. Therefore, if $R \geq 3$, the greedy algorithm becomes advantageous over the full search in terms of computational complexity. This gain will be huge when $R$ is 
large. For example, when $R=8$ which is used in our simulations, the full search considers 255 combinations while the greedy algorithm considers 36 combinations even in the worst case.

Let us compare the overall complexity roughly. We denote the complexity of the optimal numerical search by $\mathcal{F}_{\text {opt }}$, and that of our selection based method by $\mathcal{F}_{\text {sel }}$. If the grid size (interval) used by optimal numerical search is $\Delta$, the total number of grid points is $x_{k}^{\max } / \Delta$ for each relay. Thus $\mathcal{F}_{\text {opt }}=O\left(\left(x_{k}^{\max } / \Delta\right)^{R}\right)$.

On the other hand, the selection process even for full search has the relay combination number which is proportional to $\mathcal{F}_{\text {sel }}=O\left(2^{R}\right)$. Thus it is obvious that we have $\mathcal{F}_{\text {opt }} \gg \mathcal{F}_{\text {sel }}$ even if we use the well-developed optimization tools for optimal solution. This complexity gap will be larger when the grid is sufficiently dense and/or $R$ is large. Furthermore, if we combine the selection process with a greedy algorithm, the complexity reduction can be much greater.

\section{Simulation results}

In this section, we compare the proposed power allocation methods with the optimal (ex-haustive) method and two existing power allocation methods. In all simulations, we use $E_{s}=E_{r}$, and the noise variance is $\sigma_{R}^{2}=\sigma_{D}^{2}=1$. 'Exhaustive' means the power gains optimally found by a numerical method. 'Uniform' and 'Conventional' use the power allocation methods described in the section of inter-relay power allocation. The above three methods use all the relays since relay selection is not used. 'FullSearch' and 'Greedy' are the proposed relay selection based approaches.

Figures 2 and 3 shows the performance of various power allocation methods in terms of average capacity. Figure 2 is in linear scale for the $y$-axis, and a log scale is used for the $y$-axis in Figure 3 to see the difference clearly in the low SNR regime, It is observed that the selection based methods perform similarly to the optimal (exhaustive) method. When the solution for power allocation problem can not be easily derived in a closed

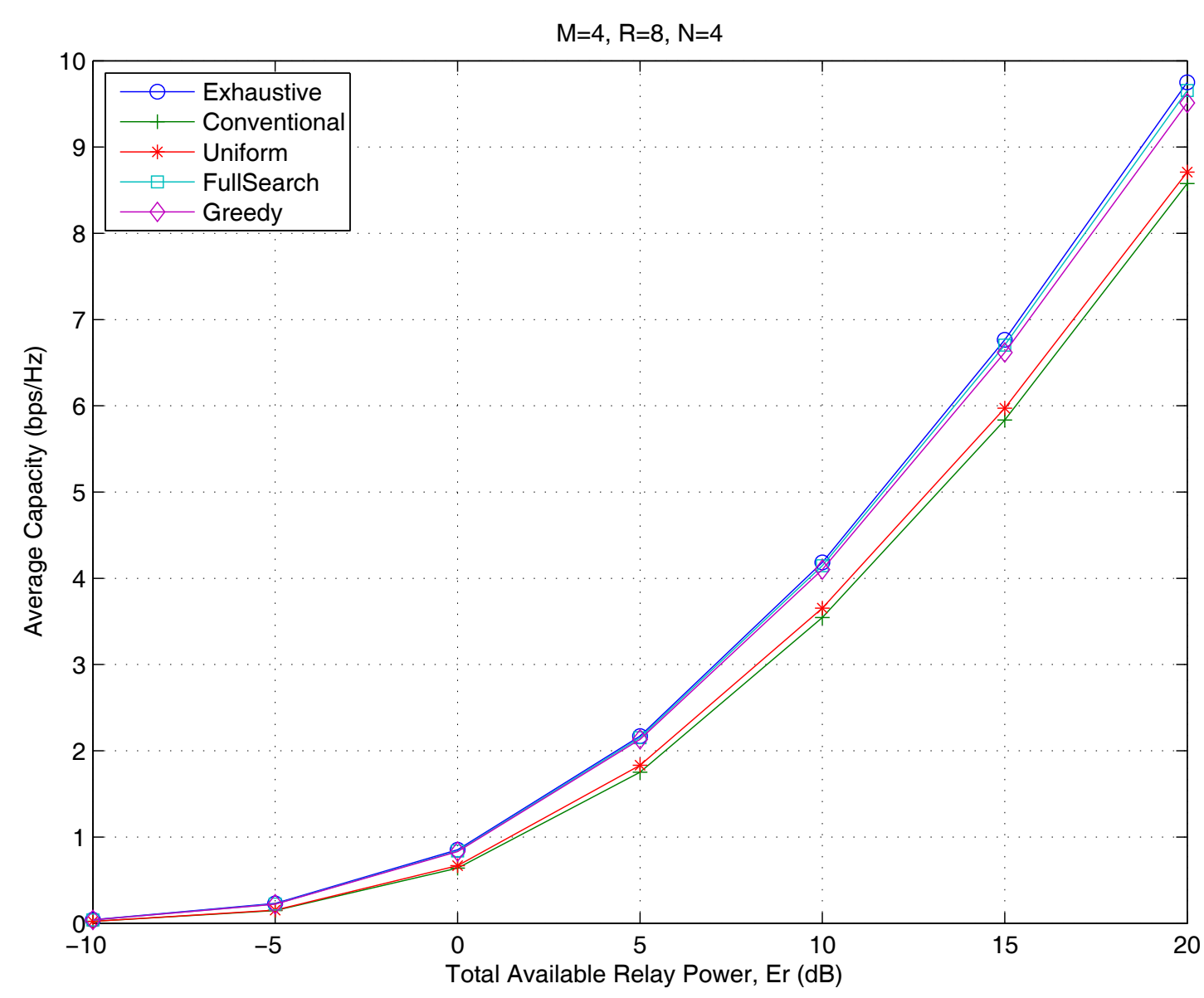

Figure 2 Average capacity versus total relay power, $E_{r}(\mathrm{~dB})$ when $M=4, R=8$, and $N=4$ (linear scale). 


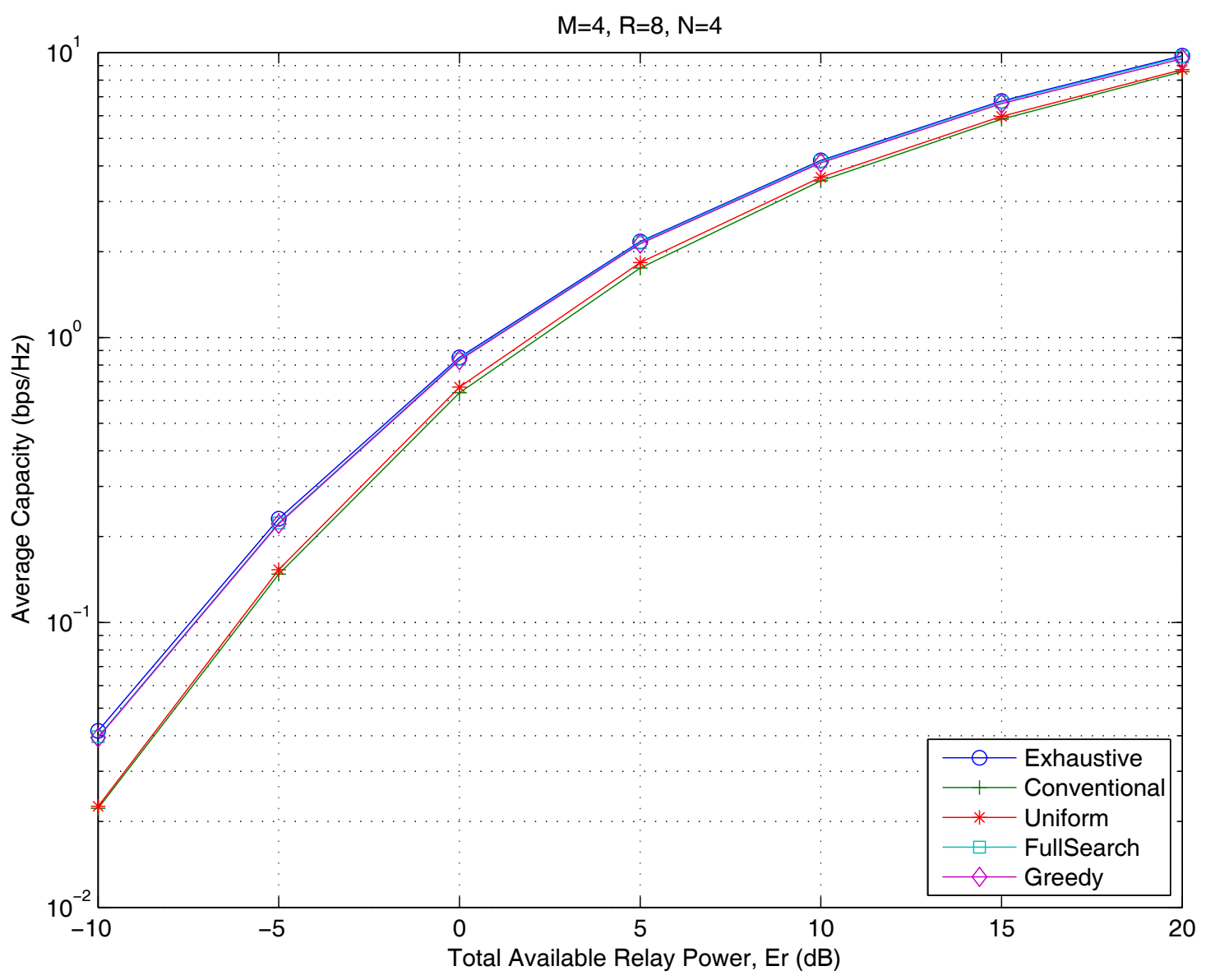

Figure 3 Average capacity versus total relay power, $E_{r}(\mathrm{~dB})$ when $M=4, R=8$, and $N=4$ (log scale).

form solution, high SNR assumption or an asymptotical approach is usually used in the literature. These tend to deviate from the optimal solution especially in the low SNR regime. However, it is interesting to note that our proposed selection techniques which are 'FullSearch' and 'Greedy' have comparable performance in all SNR regimes.

Let us consider the performance difference between the uniform gain allocation $\mathbf{g}_{\text {Unif. }}$ and the conventional power allocation $\mathbf{G}_{\text {Conv. }}$. To simplify the analysis, we focus on $g_{1}$. The gain factors are then

$$
\begin{aligned}
& \left(g_{1}\right)_{\text {Conv. }}=\sqrt{\frac{E_{r} / R}{\frac{E_{s}}{M}\left|\mathbf{h}_{11}^{T}\right|^{2}+\sigma_{R}^{2}}}, \\
& \left(g_{1}\right)_{\text {Unif. }}=\sqrt{\frac{E_{r}}{\sum_{i}\left(\frac{E_{s}}{M}\left|\mathbf{h}_{1 i}^{T}\right|^{2}+\sigma_{R}^{2}\right)}} .
\end{aligned}
$$

If it is assumed that $\left|\mathbf{h}_{11}^{T}\right|^{2}$ is the largest among all rows, that means the first hop channel to the first relay is very good. In this case, it is intuitively better to allocate more power for this relay. However, the conventional case allocates less power than the uniform case since $R \cdot\left|\mathbf{h}_{11}\right|^{2} \geq \sum_{i}\left|\mathbf{h}_{1 i}\right|^{2}$. Therefore, the uniform method performs slightly better than the conventional method. This is why we use the uniform gain allocation instead of the conventional power allocation in the proposed relay selection based approach.

In order to show the results for more general antenna configurations, we show the performance comparison with respect to the number of relays in Figure 4, where we use $E_{r}=20 \mathrm{~dB}$ to show the performance difference more clearly. We fix $M=3$ and $N=6$. The proposed methods have similar performance to the optimal (exhaustive) case for all $R$ values. The performance of the two proposed methods begin to deviate from the exhaustive method slightly as the number of relays gets 


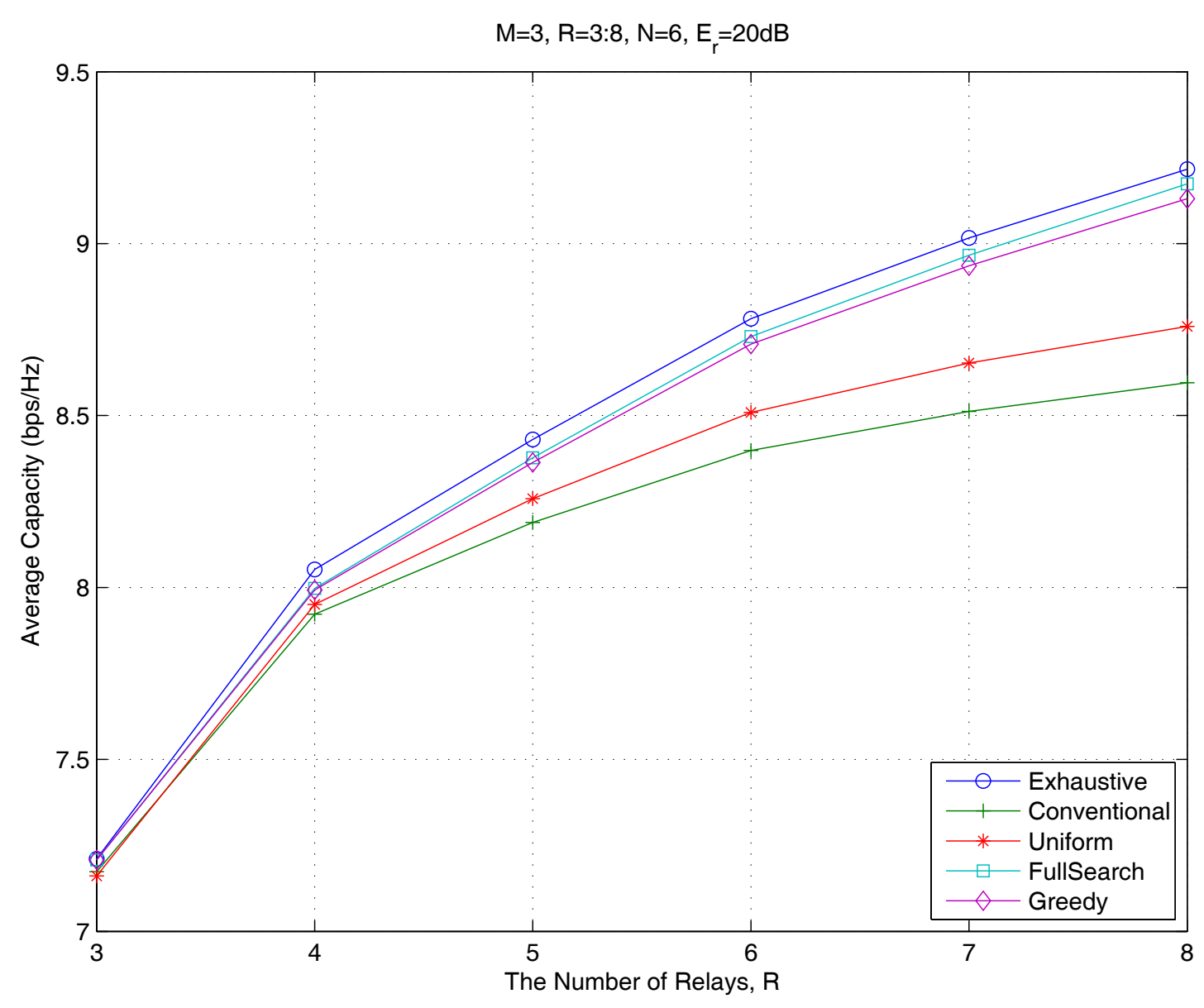

Figure 4 Average capacity versus the number of relays, $R$ when $M=3, N=6$, and $E_{r}=20 \mathrm{~dB}$.

larger. Note that the deviation is less than $0.1 \mathrm{bps} / \mathrm{Hz}$ at $R=8$. But the reduction of feedback overhead and computational complexity will be more significant as $R$ gets larger, so the proposed methods may still be attractive in practice. Figure 4 also shows that if we remove the relays in bad condition and allocate more power to the other remaining relays, the performance can be improved when uniform gain allocation is used. It is also observed that the incremental greedy search algorithm has negligible performance loss compared to the full search case.

\section{Conclusions}

In this article, we proposed a relay selection based approach for inter-relay power allocation problem. Since it is difficult to obtain a closed form solution for the problem, we propose a low-complexity technique based on relay selection using uniform gain allocation, which has near-optimal performance in terms of capacity. We also combine with the proposed method with an incremental greedy search algorithm in order to further reduce the search complexity for the relay selection. Simulations show that the proposed methods for relay power allocation have performance close to the optimal (exhaustive) power allocation in terms of average capacity. The proposed methods appear to be promising in terms of computational complexity and capacity performance.

\section{Endnote}

${ }^{a}$ If we consider the complex valued gain, the performance can be improved by adjusting the phase with extra processing. When a complex number is treated as two real values (magnitude and phase), the feedback overhead (as will be discussed) may overshadow the benefit of power allocation. Thus we focus on the real valued power allocation problem in this article.

\section{Acknowledgements}

This research was supported in part by the Basic Science Research Programs (KRF-2008-314-D00287, 2010-0013397), Mid-career Researcher Program (20100027155) through the NRF funded by the MEST, Seoul R\&BD Program 
(JP091007, 0423-20090051), the KETEP Grant (2011T100100151), the INMAC, and BK21.

\section{Competing interests}

The authors declare that they have no competing interests.

Received: 9 July 2011 Accepted: 30 May 2012 Published: 30 May 2012

\section{References}

1. EC van der Meulen, Three-terminal communication channels. Adv Appl Probl. 3, 120-154 (1971). doi:10.2307/1426331

2. T Cover, AE Gamal, Capacity theorems for the relay channel. IEEE Trans Inf Theory. 25(5), 572-584 (1979). doi:10.1109/TIT.1979.1056084

3. JN Laneman, DNC Tse, GW Wornell, Cooperative diversity in wireless networks: efficient protocols and outage behavior. IEEE Trans Inf Theory. 50(12), 3062-3080 (2004). doi:10.1109/TT.2004.838089

4. JN Laneman, GW Wornell, Distributed space-time-coded protocols for exploiting co-operative diversity in wireless networks. IEEE Trans Inf Theory. 49(10), 2415-2425 (2003). doi:10.1109/TIT.2003.817829

5. A Nosratinia, TE Hunter, A Hedayat, Cooperative communication in wireless networks. IEEE Commun Mag. 42(10), 74-80 (2004). doi:10.1109/ MCOM.2004.1341264

6. A Sendonaris, E Erkip, B Aazhang, User cooperation diversity-Part I, II. IEEE Trans Commun. 51(11), 1927-1948 (2003). doi:10.1109/TCOMM.2003.818096

7. MO Hasna, MS Alouini, Optimal power allocation for relayed transmissions over Rayleigh-fading channels. IEEE Trans Wirel Commun. 3(6), 1999-2004 (2004). doi:10.1109/TWC.2004.833447

8. X Deng, AM Haimovich, power allcoation for cooperative relaying in wireless networks. IEEE Commun Lett. 9(11), 994-996 (2005). doi:10.1109/ LCOMM.2005.11012

9. Y Zhao, R Adve, TJ Lim, Improving amplify-and-forward relay networks: optimal power allocation versus selection. IEEE Trans Wirel Commun. 6(8), 3114-3123 (2007)

10. N Khajehnouri, AH Sayed, Distributed MMSE relay strategies for wireless sensor networks. IEEE Trans Signal Process. 55(7), 3336-3348 (2007)

11. W Guan, H Luo, Joint MMSE transceiver design in non-regenerative MIMO relay sys-tems. IEEE Commun Lett. 12(7), 517-519 (2008)

12. R Krishna, Z Xiong, S Lambotharan, A cooperative MMSE relay strategy for wireless sensor networks. IEEE Signal Process Lett. 15, 549-552 (2008)

13. H Muhaidat, M Uysal, Cooperative diversity with multiple-antenna nodes in fading relay channels. IEEE Trans Wirel Commun. 7(8), 3036-3046 (2008)

14. O Muñoz-Medina, J Vidal, A Agustín, Linear transceiver design in nonregenerative relays with channel state information. IEEE Trans Signal Process. 55(6), 2593-2604 (2007)

15. X Tang, Y Hua, Optimal design of non-regenerative MIMO wireless relays. IEEE Trans Wirel Commun. 6(4), 1398-1407 (2007)

16. AS Behbahani, R Merched, AM Eltawil, Optimizations of a MIMO relay network. IEEE Trans Signal Process. 56(10), 5062-5073 (2008)

17. H Shi, T Abe, T Asai, H Yoshino, Relaying schemes using matrix triangularization for MIMO wireless networks. IEEE Trans Commun. 55(9), 1683-1688 (2007)

18. B Maham, A Hjørungnes, Opportunistic relaying through amplify-andforward distributed space-time codes with partial statistical CSI at relays, in Proc IEEE Allerton Conference on Communication, Control an Computing, Monticello, IL, pp. 1004-1008 (2008)

19. B Maham, A Hjørungnes, Opportunistic relaying for MIMO amplify-andforward cooperative networks. Wirel Pers Commun 1-25 http://dx.doi.org/ 10.1007/s11277-011-0499-9 (2012)

20. L Sun, MR MCKay, Opportunistic relaying for MIMO wireless communication: relay selection and capacity scaling laws. IEEE Trans Wirel Commun. 10(6), 1786-1797 (2011)

21. TM Cover, JA Thomas, Elements of Information Theory (Wiley, New York, 1991)

doi:10.1186/1687-1499-2012-183

Cite this article as: Bae and Lee: Selection based inter-relay power allocation for multiple AF single-antenna relays. EURASIP Journal on Wireless Communications and Networking 2012 2012:183.

\section{Submit your manuscript to a SpringerOpen ${ }^{\mathcal{O}}$ journal and benefit from:}

- Convenient online submission

- Rigorous peer review

- Immediate publication on acceptance

- Open access: articles freely available online

- High visibility within the field

- Retaining the copyright to your article

Submit your next manuscript at $\gg$ springeropen.com 